\title{
Reducing Mental Health Stigma in Primary Healthcare Workers in a Rural Chinese County: a Contact-based Family-centric Approach
}

\author{
Selinda Qiu \\ University of Pennsylvania \\ Tian-Ming Zhang \\ Shanghai University \\ Yuer Deng \\ University of Pennsylvania \\ Mao-Sheng Ran \\ Hong Kong University \\ Yin-Ling Irene Wong \\ University of Pennsylvania
}

An-Li Wang ( $\sim$ anliwang@gmail.com )

Icahn School of Medicine at Mount Sinai https://orcid.org/0000-0003-0938-5157

\section{Research}

Keywords: Stigma reduction, Mental-health related stigma, Healthcare workers, Contact-based familycentric training, Rural Chinese community

Posted Date: August 27th, 2021

DOl: https://doi.org/10.21203/rs.3.rs-841056/v1

License: (c) (i) This work is licensed under a Creative Commons Attribution 4.0 International License. Read Full License 


\section{Abstract}

Background: Mental health-related stigma is a key deterrent to help-seeking in people with mental illness. However, effective stigma reduction interventions are sparse, let alone ones adapted to the cultural context of mental health systems. The aims of the present study were to demonstrate the feasibility and to evaluate the impact of a culturally adapted brief contact-based family-centric training program on reducing mental health-related stigma in primary healthcare workers (PHW) in a Chinese rural county.

Methods: The one-day stigma reduction training program was developed based on the Opening Minds Anti-Stigma Initiative. The program included didactic lectures, case studies (two audio-taped testimonies of patient-caregiver dyads) and communication skills training (role-playing). Ten township-level and ten village-level PHW participated in separate one-day training sessions. Mental health knowledge, stigmatizing attitudes and behaviors, and provider empathy were measured before and after the training.

Results: The intervention was favorably rated and considered acceptable among PHW. Compared to measures at baseline, participants' stigmatizing attitudes reduced, their willingness to engage in relationship with people with mental illness increased, and their empathetic response toward patients improved post-intervention.

Conclusions: Social contact-based family-centric training is a promising and feasible method of reducing stigma among rural Chinese PHW.

\section{Background}

Mental health-related stigma is a key deterrent to help-seeking in people with mental illness (1). In Chinese culture, traditional values rooted in Confucianism, Taoism, and Buddhism endorse the primacy of families as the core unit providing sustenance and social support based on the ethos of interdependence among family members (2). Concurrently, Chinese health beliefs stigmatize people with mental illness and their families because psychiatric disorders are considered punishment for their collective moral failings and for their ancestors' transgressions. Thus, concealment and secrecy are common coping strategies used by Chinese families to mediate social rejection, and the propensity of seeking care is hampered by perceived stigma experienced by people with mental illness and courtesy stigma by family members (3).

In China, professional mental health services are concentrated in urban centers, leading to rural-urban disparities in contact coverage for different diagnoses, availability of psychiatric beds, and proportion of people with mental illness seeking outpatient treatment (4). The 2004 Continuing Management and Intervention Program for Psychoses has facilitated the expansion of local community-based mental health service networks in rural China (5). In rural communities, township- and village-level primary healthcare workers (PHW) are employed under the supervision of psychiatrists or physicians of other specialties to "manage" community-dwelling people with severe mental disorders. The responsibilities of PHW include conducting regular wellness visits, monitoring treatment and medication adherence, and 
facilitating annual physical examinations for patients. These PHW have limited training in mental health services delivery and may hold stigmatizing attitudes toward patients and their families because traditional norms and values are more ingrained in rural communities than their urban counterparts (6).

In this paper, we reported results of a brief contact-based family-centric training program aimed to reduce mental health-related stigma held by rural, primary healthcare workers. Despite the pervasiveness of stigma and low treatment rate of psychiatric patients, stigma reduction programs targeting healthcare providers in China are scant and most are implemented in urban areas (6). The design of this one-day program was informed by the Opening Minds Anti-Stigma Initiative (OM Initiative) in Canada. The OM Initiative used contact-based educational strategies where target audiences heard first-person testimony of recovery from a trained speaker with lived experience of mental illness (7). Three ingredients of the OM Initiative for healthcare providers were incorporated in the current program: 1) providing social contact through personal testimony, 2) emphasizing recovery and hope in program messaging; and 3) facilitating providers' behavioral change through teaching skills (8). The research team adapted the anti-stigma intervention with due consideration of the unique socio-cultural characteristics of the study community.

\section{Methods}

Twenty PHW were recruited by availability sampling through a county psychiatric hospital liaison office in southwestern China. The stigma reduction program was designed and delivered by a China-U.S. team of bilingual mental health researchers and practitioners. The study protocol was approved, respectively, by the human research ethics committees of the two collaborating universities. All participates signed informed consent.

As Chinese rural households are embedded in close-knit webs of kinship and village networks, providing in-person testimony of patients and family members about individuals' lived experiences of stigma and discrimination while maintaining social discretion was deemed unfeasible. As such, the research team conducted 32 photo-elicitation interviews of 16 patient-caregiver dyads in the study site prior to launching the training program to generate narratives and storylines (9). Interview transcripts of two dyads with the highest scores in recovery and hope as rated by the research team were developed into two audio-taped vignettes presented by voice actors.

In addition, four focus groups were conducted with 36 healthcare providers to explore their attitudes toward mental illness and patient care. Providers identified professional knowledge (e.g. diagnosis and medication) and communication skills with patients, families, and other stakeholders as their top training priorities (6). An educational lecture on community-based case management and prevalence, diagnosis, and treatment of schizophrenia, as well as communication training through role-playing were therefore included in the program. The lecture and vignettes focused on schizophrenia, the most common psychiatric diagnosis in the county mental health system.

Figure 1 illustrates a workflow of the training protocol. The program began with a didactic lecture, followed by a case study (see the bottom panel of Fig. 1 for the life-history calendar of the vignette) with 
participants completing workbook exercises and participating in an interactive brainstorming session. The program continued with skills training centering on three scenarios: 1) providing emotional support to the caregiver at illness onset; 2) reaching out to the patient who did not consider himself to have mental illness; and 3) continuing support for the family when the patient recovered. The program concluded with a second vignette reinforcing the message of recovery and hope. English version of the two vignettes are included as supplementary materials.

Participants' mental health knowledge, attitudes and behaviors toward people with mental illness, and empathy for psychiatric patients were assessed before and after the program. Mental health knowledge was assessed with the Mental Health Knowledge Schedule (MAKS). Participants' attitudes toward mental illness and psychiatry was measured with the Mental Illness: Clinicians' Attitudes Scale (MICA).

Participants' intended mental health-related behavioral discrimination was measured by the Reported and Intended Behaviors Scale (RIBS). The Social Distance Scale (SDS), a vignette-based measure, assessed the social distance participants intended to put between themselves and a hypothetical individual experiencing psychotic symptoms. The Jefferson Scale of Physician Empathy-Health Professional version (JSE-HP) assessed participants' empathetic reactions to patient care. All five measures have shown high reliability and validity, and have been translated and used in Chinese populations $(5,10,11$, 12). In addition, an 11-item evaluation form assessed participants' perceptions on the accomplishment of training objectives, quality of training experience, and relevance of training to participants' work.

Paired-sample t-tests were conducted to examine the changes in the outcome measures before and after the training program. Mean scores were computed to assess the acceptability of the intervention using the training evaluation form.

\section{Results}

Two one-day programs using the same training protocol were implemented for township PHW and village PHW, respectively. Ten township PHW and ten village PHW (90\% female, $37.95 \pm 8.31$ years old, Mean \pm SD) participated in the study. Ten (50\%) of the PHW had a high school diploma, nine (45\%) had an associate degree, and one (5\%) had a college degree. Eleven (55\%) had less than five years of experience working with psychiatric patients, and one $(5 \%)$ had more than ten years of relevant work experience.

No significant differences were found between township PHW and village PHW in all outcome measures at baseline. After the completion of the program, we found that attitudes toward mental illness and psychiatry significantly improved (MICA, $p=.018$ ), suggesting a positive attitude change. The SDS mean score was significantly lower $(p=.018)$, indicating the participants were more willing to engage in relationships of varying intimacy with a person with mental illness post-training. The JSE-HP mean score was significantly enhanced $(p=.022)$, indicating improved level of participants' empathetic response toward patient care. There was no significant change in mental health knowledge (MAKS, $p=1.000$ ) and intended behaviors toward people with mental illness (RIBS, $p=.150)$. 
The training program was considered acceptable for both township and village providers. On a five-point scale with five indicating the highest favorable rating, the mean scores of the 11 evaluation items range from 4.6 to 4.8 (SD from \pm 0.41 to \pm 0.75 ). Participants found the materials covered to be relevant and helpful for their work, and felt that the trainers were knowledgeable and well prepared. While participants were largely satisfied with the program format and the way the content was delivered, they cited the amount of participation and interaction, and time allotted for skills training as areas for improvement.

\section{Discussion}

Our findings suggest that a brief contact-based family-centric stigma reduction program promotes positive changes in mental health-related sigma in Chinese primary healthcare workers. A strength of this pilot is the adaptation of the ingredients of an anti-stigma intervention tested and disseminated in Canada to rural China (8). Research shows that cultural adaptation of psychosocial interventions increases their effectiveness, but few stigma reduction programs report methods for adaptation (13). Specific to the current study, conducting photo-elicitation interviewing in the study county enabled the infusion of site-specific socio-cultural characteristics into the design of family-centric messaging.

Aside from maintaining patient privacy, using audio-taped vignettes as a way to facilitate indirect social contact is culturally appropriate because direct in-person exchanges between psychiatric patients and healthcare providers are likely to create uneasiness in patient-provider interactions in a training setting. PHW act as proxies of China's mental health system, delegated with the responsibility of managing the risks psychiatric patients pose to their own safety and welfare, and the security of the general public (14, 15). Often, providers and family members make treatment decisions independent of patients' wishes, constraining patients' liberty of action as psychiatric patients are considered to be vulnerable dependents with compromised decision-making capacity (14). It is worth noting that our program encouraged PHW to learn about the lived experience of psychiatric patients and treat them as an active agent in their own recovery rather than a passive or coerced subject.

To our knowledge, the current program is the first of its kind to also incorporate the experiences and perspectives of family members in stigma reduction targeting healthcare providers. Under the 2013 Mental Health Law, Chinese families are responsible for the care and management of communitydwelling patients and held accountable for the behaviors of their ill members (14). However, families are often the sole caregiving units for their kin with mental illness, because decades of market reform in China have eroded socialist institutions involved in the care of psychiatric patients, including work units and communes and their accompanying welfare systems (15). Our approach of developing family-centric vignettes as a key part of messaging acknowledges the indispensable role that families play in providing support to their ill relatives.

Although our findings are promising with regards to reducing stigmatizing attitudes, increasing willingness to engage in relationships with people with mental illness, and increasing provider empathy, these short-term results need to be interpreted with caution because of small sample size and the lack of 
a comparison condition. Nevertheless, the current study contributes to a limited body of research from low- and middle-income countries that examined interventions to reduce mental health-related stigma among healthcare providers (12).

\section{Conclusions}

This culturally-adapted, social-contact based intervention centered on families affected by mental illness showed promising results in reducing aspects of primary care provider stigma toward people with mental illnesses in a rural county. Future research should address the potential of facilitating long-term, sustained stigma reduction through increasing the length and frequency of training sessions and evaluating the effects of stigma reduction on treatment engagement and health outcomes of psychiatric patients.

\section{Declarations}

Ethics approval and consent to participate: Yes

Consent for publication: Yes

Availability of data and materials: Yes.

Competing interests: All authors disclose no competing interests.

Funding: Penn China Research and Engagement Fund, University of Pennsylvania

Authors' contribution: ALW and YIW conceptualized and design the study. ALW, MSR, YIW, TMZ, YD, SQ implemented the protocol and collected data. ALW, SQ and YIW analyzed, interpreted the data and drafted the manuscript. All authors commented on the manuscript.

Acknowledgements: The authors thank all the staff of the Xinjin Mental Hospital for collaboration and support. We are indebted to persons with schizophrenia and their family caregivers, and healthcare providers who participated in the design phase of the study.

\section{References}

1. Clement S, Schauman O, Graham T, Maggioni F, Evans-Lacko S, Bezborodovs N, Morgan C, Rusch N, Brown JS, Thornicroft G. What is the impact of mental health-related stigma on help-seeking? A systematic review of quantitative and qualitative studies. Psychol Med. 2015;45(1):11-27. Epub 2014/02/27. doi: 10.1017/S0033291714000129. PubMed PMID: 24569086.

2. Lam CS, Tsang HWH, Corrigan PW, Lee Y-T, Angell B, Shi K, Jin S, Larson JE. Chinese lay theory and mental illness stigma: implications for research and practices. The Journal of Rehabilitation. 2010;76:35-40. 
3. Goffman E. Stigma: Notes on the management of spoiled identity: Simon and Schuster; 2009.

4. Patel V, Xiao S, Chen H, Hanna F, Jotheeswaran AT, Luo D, Parikh R, Sharma E, Usmani S, Yu Y, Druss $B G$, Saxena $S$. The magnitude of and health system responses to the mental health treatment gap in adults in India and China. Lancet. 2016;388(10063):3074-84. Epub 2016/05/23. doi:

10.1016/S0140-6736(16)00160-4. PubMed PMID: 27209149.

5. Li J, Li J, Huang Y, Thornicroft G. Mental health training program for community mental health staff in Guangzhou, China: effects on knowledge of mental illness and stigma. International Journal of Mental Health Systems. 2014;8(1):49. Epub 2014/12/19. doi: 10.1186/1752-4458-8-49. PubMed PMID: 25520750; PMCID: PMC4268793.

6. Deng Y, Wang AL, Frasso R, Ran MS, Zhang TM, Kong D, Wong YI. Mental health-related stigma and attitudes toward patient care among providers of mental health services in a rural Chinese county. International Journal of Social Psychiatry. 2021:20764021992807. Epub 2021/02/09. doi: 10.1177/0020764021992807. PubMed PMID: 33554704.

7. Stuart H, Chen S-P, Christie R, Dobson K, Kirsh B, Knaak S, Koller M, Krupa T, Lauria-Horner B, Luong D, Modgill G, Patten SB, Pietrus M, Szeto A, Whitley R. Opening Minds in Canada: Background and Rationale. Canadian Journal of Psychiatry. 2014;59(1_suppl):8-12. doi:

$10.1177 / 070674371405901 s 04$.

8. Knaak S, Modgill G, Patten SB. Key ingredients of anti-stigma programs for health care providers: a data synthesis of evaluative studies. Canadian Journal of Psychiatry. 2014;59(10 Suppl 1):S19-26. Epub 2015/01/08. doi: 10.1177/070674371405901 s06. PubMed PMID: 25565698; PMCID: PMC4213748.

9. Harper D. Talking about pictures: A case for photo elicitation. Visual Studies. 2002;17(1):13-26. doi: 10.1080/14725860220137345.

10. Hsiao C-Y, Lu H-L, Tsai Y-F. Factors influencing mental health nurses' attitudes towards people with mental illness. International Journal of Mental Health Nursing. 2015;24(3):272-80. doi: https://doi.org/10.1111/inm.12129.

11. Li J, Li J, Thornicroft G, Yang H, Chen W, Huang Y. Training community mental health staff in Guangzhou, China: evaluation of the effect of a new training model. BMC Psychiatry. 2015;15:263. Epub 2015/10/28. doi: 10.1186/s12888-015-0660-1. PubMed PMID: 26503370; PMCID: PMC4620640.

12. Fang Q, Zhang TM, Wong YLI, Yau YY, Li XH, Li J, Chui CHK, Tse S, Chan CLW, Chen EYH, Ran MS. The mediating role of knowledge on the contact and stigma of mental illness in Hong Kong. International Journal of Social Psychiatry. 2020; 1-11. doi:10.1177/0020764020975792.

13. Heim E, Kohrt B, Koschorke M, Milenova M, Thornicroft G. Reducing mental health-related stigma in primary health care settings in low-and middle-income countries: a systematic review. Epidemiology and psychiatric sciences. 2020;29(e3):1-10. doi: https://doi.org/10.1017/S2045796018000458

14. Ma Z. Biopolitical Paternalism and Its Maternal Supplements: Kinship Correlates of Community Mental Health Governance in China. Cultural Anthropology. 2020;35(2):290--316. doi: 
http://dx.doi.org/10.14506/ca35.2.09. PubMed PMID: 2442625922.

15. Ma Z. Promises and perils of Guan. Medicine Anthropology Theory. 2020;7(2):150-74.

\section{Supplementary Materials}

Supplementary Materials are not available with this version.

\section{Figures}

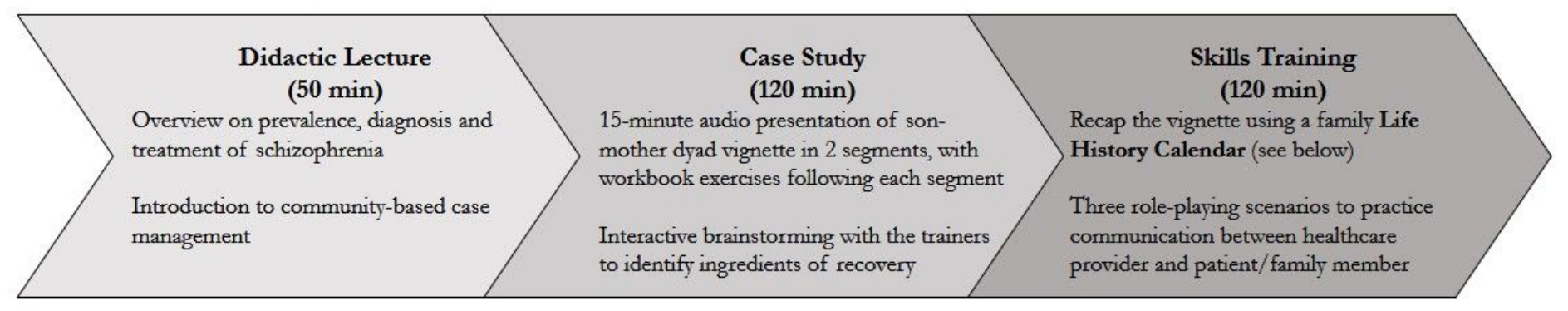

Life History Calendar (Son-Mother Dyad)

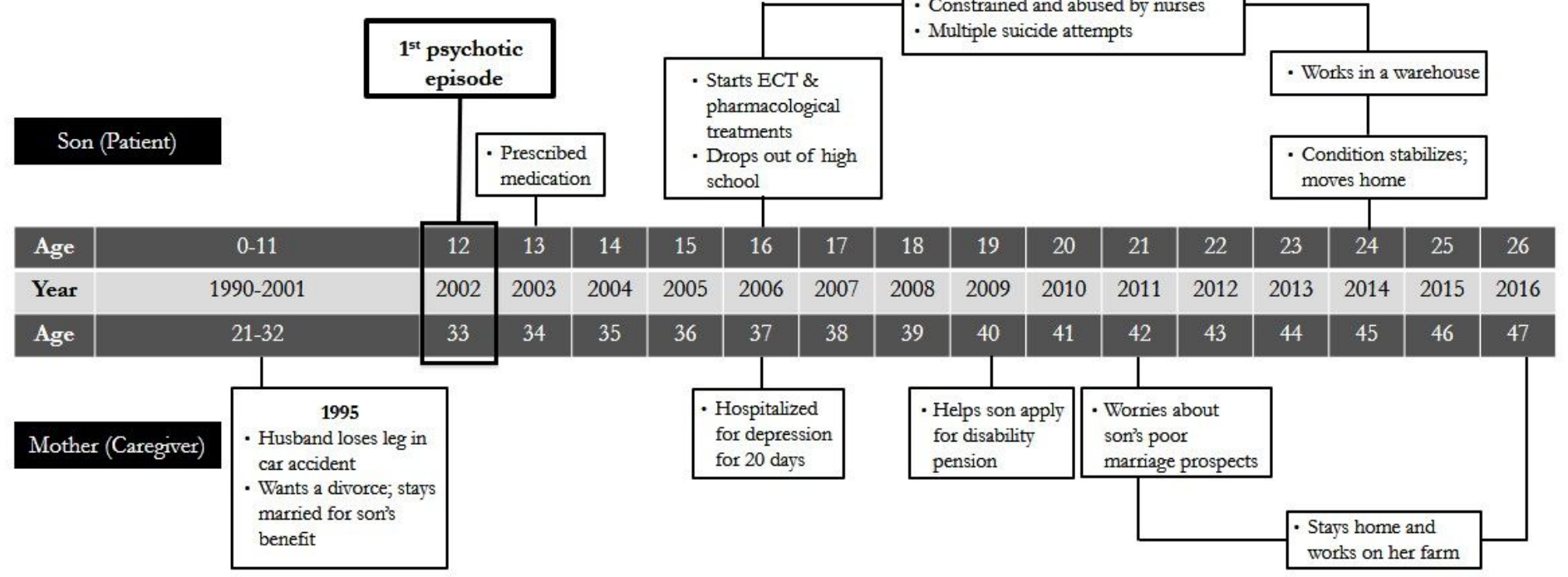

\section{Figure 1}

A workflow of the contact-based family-centric training program 